\title{
Evolution of the First Stars: the major role of rotation for mixing and mass loss
}

\author{
André Maeder, Georges Meynet, and Sylvia Ekström \\ Geneva Observatory, CH-1290 Sauverny, Switzerland \\ email:andre.maeder@obs.unige.ch \\ email: georges.meynet@obs.unige.ch \\ email: sylvia.ekstrom@obs.unige.ch
}

\begin{abstract}
We show that rotation plays a major role for very low metallicity stars, even if the distribution of angular velocities $\Omega$ with respect to critical values is the same as at solar $Z$. The internal gradients of $\Omega$ are much larger at lower metallicity $Z$, which enhance internal mixing and give rise to N-enrichments. Low $Z$ stars easily reach break-up during MS evolution and lose mass. They also lose mass as red giants or supergiants due to their surface enrichments in heavy elements. The winds of low $Z$ stars make peculiar contributions to the chemical yields. We suggest that the helium rich blue Main Sequence (bMS) of $\omega$ Centauri bears the signature of such enrichments by the stellar winds of rotating stars in the first stellar generations.
\end{abstract}

Keywords. Stars: abundances, stars: rotation, stars: mass loss, stars: AGB and post-AGB

\section{Introduction}

At solar metallicity, the effects of rotation, although significant, are rather limited in size. The situation is quite different at lower metallicity $Z$, for the physical reasons given in Sect. 2. There is a suggestion that stars may rotate faster at lower $Z$ (Maeder, Grebel \& Mermilliod 1999). The suggestion is based on the increasing fraction of Be stars from the Milky Way, to the LMC and SMC. However, even if the distribution of the ratios $\Omega / \Omega_{\mathrm{c}}$ of the angular velocity to the break-up velocity is the same at the various metallicities, rotation is nevertheless a dominant effect at lower $Z$, with implications for the early chemical evolution of galaxies.

\section{Rotational effects at very low $Z$}

At $Z=0$, the stellar radii $R$ are about a factor of 4 smaller than at solar metallicity. This is illustrated by Fig. 1, which compares the tracks at $Z=0$ and $Z=0.02$ in the HR diagram. The smaller radii at lower $Z$ result from the lower opacities. The compactness of $Z=0$ stars is a key property, which has many effects in relation with stellar rotation. Below we describe the main effects due to rotation at zero and very low metallicities (cf. papers I-XII on star models with rotation, e.g. Meynet \& Maeder 2002; Meynet, Ekström \& Maeder 2005).

(a) More mixing: At $Z$ lower than solar, there is much more mixing predicted (Maeder \& Meynet 2001) and observed (Venn \& Przybilla 2004). This is due to several physical reasons: 1.- The smaller radii reduce the mixing timescales. For a diffusion coefficient $D$, the diffusion timescales behave like $\tau \sim R^{2} / D$, so that radii smaller by a factor of 4 imply diffusion timescales shorter by a factor of 16 . 2.- Being more compact, the lower $Z$ stars have much steeper internal gradients of $\Omega$, as illustrated by Fig. 2 . This means stronger shears and in turn more chemical mixing, since shear turbulence is the main effect driving the internal mixing (Zahn 1992). 3.- There is a third effect which 


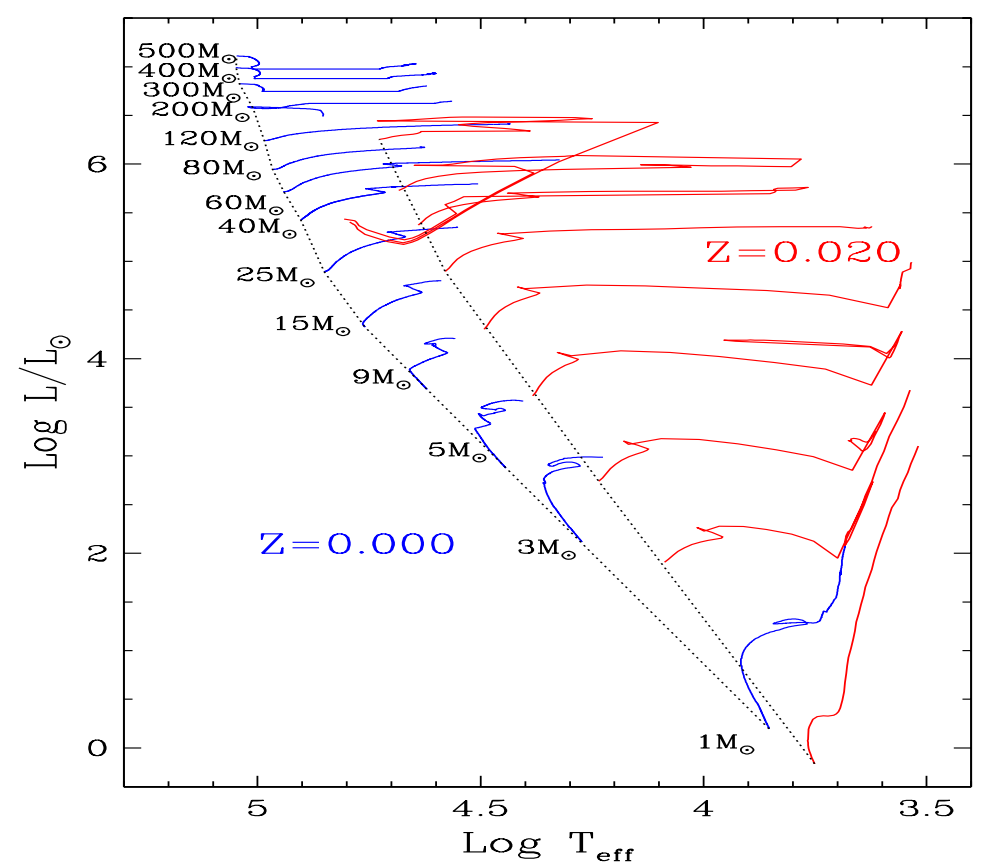

Figure 1. Evolutionary tracks in the HR diagram at $Z=0$ and $Z=0.02$, (Feijoo 1999).

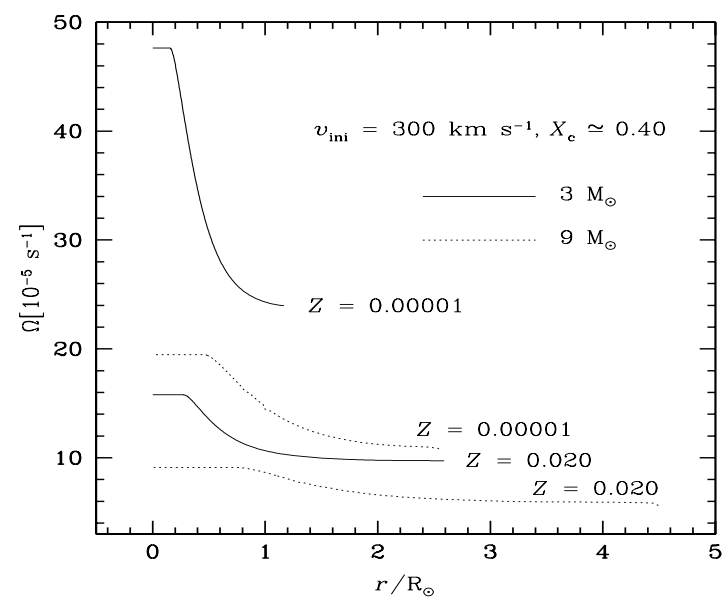

Figure 2. The different internal $\Omega$-gradients in stars of various masses and $Z$.

favours mixing at lower $Z$ : the differences in meridional circulation. The main driving term of meridional circulation behaves like (Maeder \& Zahn 1998)

$$
E_{\Omega} \sim \frac{\frac{\Omega^{2} r^{3}}{G M}}{\left(1-\frac{\Omega^{2}}{2 \pi G \bar{\rho}_{\text {int }}}\right)} .
$$

The circulation grows with the ratio of centrifugal force to gravity. At the denominator of the fraction, there is a term depending on the average density $\bar{\rho}_{\text {int }}$ interior to the level considered. At solar $Z$, the relatively low density in the outer layers makes the 


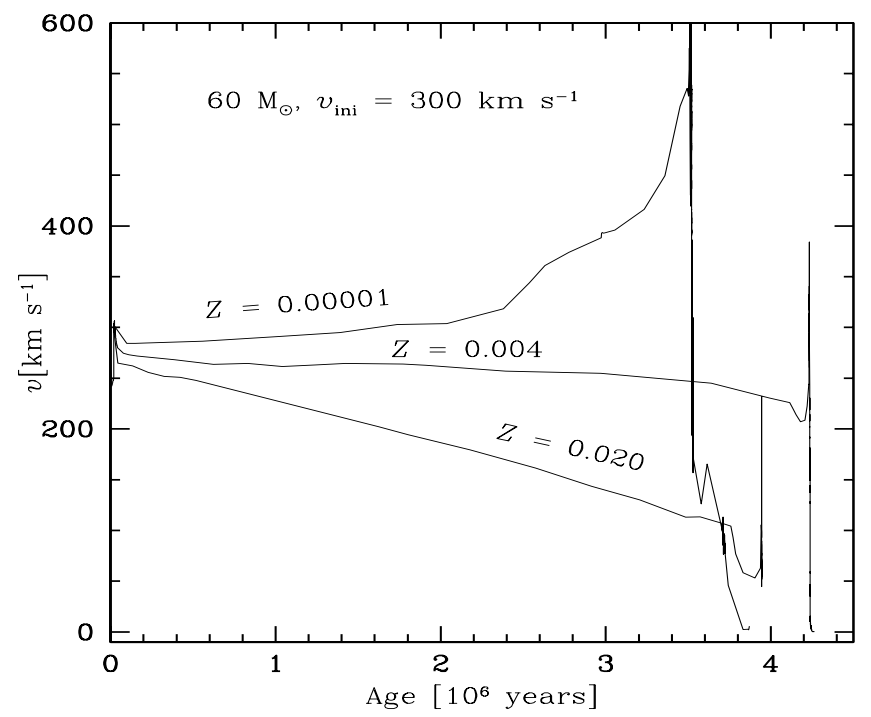

Figure 3. Evolution of the rotation velocities of $60 \mathrm{M}_{\odot}$ stars with various $Z$.

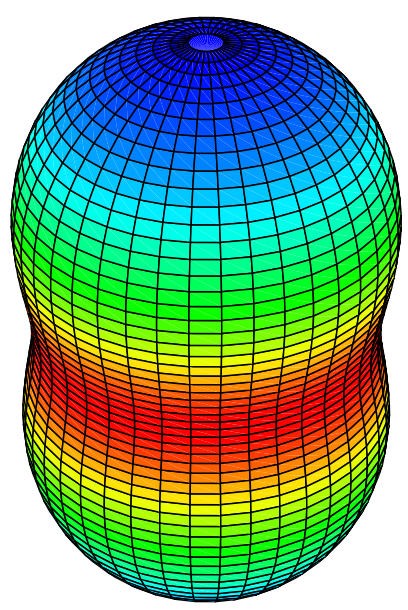

Figure 4. Distribution of the mass loss rates around a rotating hot $\operatorname{star}\left(T_{\text {eff }}=30000 \mathrm{~K}\right)$ with electron scattering opacity, (the polar axis is vertical). The length of the vector from the center to the peanut-shaped surface is proportional to the amount of mass loss in a given direction.

denominator of the above expression negative, thus there is a large inverse circulation cell (we call it the Gratton-Öpik cell) descending along the polar axis and rising in the equatorial plane. This Gratton-Öpik cell lies above the usual cell turning the other way around. It brings angular momentum outwards and thus reduces the $\Omega$-gradients at solar $Z$. At lower $Z$, the higher density reduces, or even suppresses, this outer cell and this 


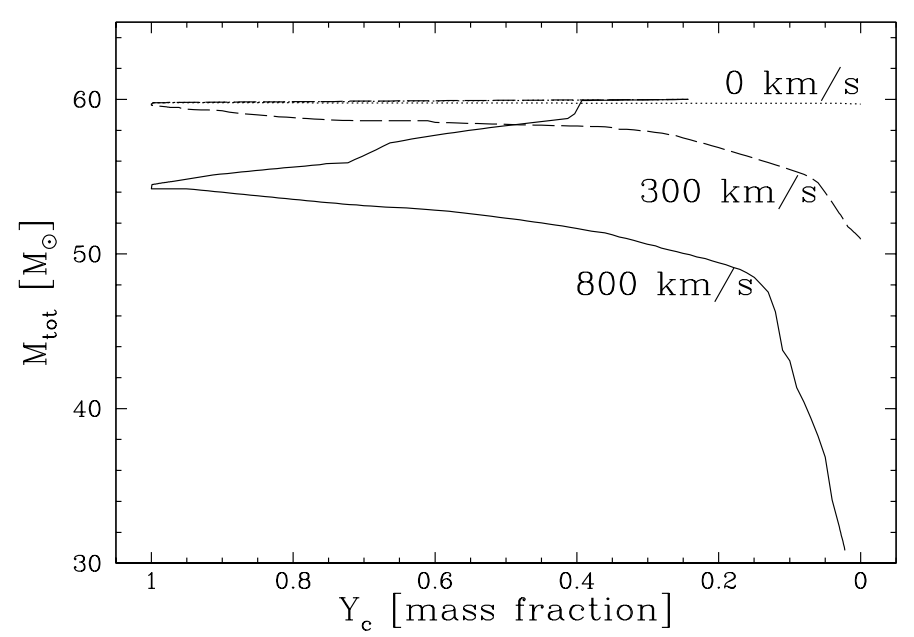

Figure 5. Evolution of the mass models of an initial $60 \mathrm{M}_{\odot}$ star at $Z=10^{-8}$ as a function of the central helium mass fraction $Y_{\mathrm{c}}$ for different initial rotation velocities. Caution: evolution starts at $Y_{\mathrm{c}}=0.24$ at the beginning of the MS phase, then reaches 1.0 at the end of the MS phase, then moves to $Y_{\mathrm{c}}=0$ at the end of the He-burning phase.

makes the $\Omega$-gradients steeper. As a result of these 3 effects, there is more mixing in rotating stars at lower $Z$. Indeed, several observations of $\mathrm{O}$-stars and supergiants in the LMC and SMC confirm the presence of more mixing than in the Galaxy. For example, Venn \& Przybilla (2004) found large relative $N / H$-enrichments for A-type supergiants in the SMC, reaching a factor of 20-40 while in the Milky Way the $N / H$-enrichments amount to a factor of 2 to 3 .

(b) Rotation keeps faster during evolution: At lower $Z$, the stellar winds are weaker and the losses of mass and angular momentum are smaller. This favours larger rotational velocities during further evolution (Fig. 3). In addition, the stellar distortion due to rotation implies hotter polar caps and cooler equatorial regions, according to the von Zeipel theorem. Thus, the radiative winds are stronger at the pole and weaker at the equator (Maeder 2002), as illustrated in Fig. 4. This is true if electron scattering dominates, which is the case at $Z=0$ for stars with masses $\geqslant 5 \mathrm{M}_{\odot}$. Polar mass loss removes mass, but little angular momentum. This also favours higher rotation velocities during further evolution and the stars more easily reach break-up, as illustrated in Fig. 1 by Ekström et al. in these Proceedings. We note that for stars with $T_{\text {eff }} \leqslant 25000 \mathrm{~K}$, the higher opacities lead to the formation of an equatorial ring around rotating stars (Maeder 2002). In this case, typically of models at solar $Z$, the stars lose a lot of angular momentum.

(c) Possibility of heavy mass loss at very low $Z$ : Three different effects contribute to mass loss in low $Z$ stars. First, low $Z$ stars easily reach break-up during the MS phase (Fig. 3) and lose some mass. As illustrated in Fig. 5, the amount of mass lost during the MS phase is moderate. The second effect occurs in the red giant or supergiant phase. There, the stars have considerable enhancements in CNO elements (cf. Fig. 6) so that their surface metallicities may increase by a factor up to $10^{6}$, which leads to significant stellar winds. Third, when red giants and supergiants move back to the blue, the contraction of the external convective zone again brings the star to break-up with a very large mass loss. From Fig. 5 we see that a $60 \mathrm{M}_{\odot}$ star with $Z=10^{-8}$ and an initial velocity of $800 \mathrm{~km} \mathrm{~s}^{-1}$ loses on the whole about half of its initial mass before the end of 


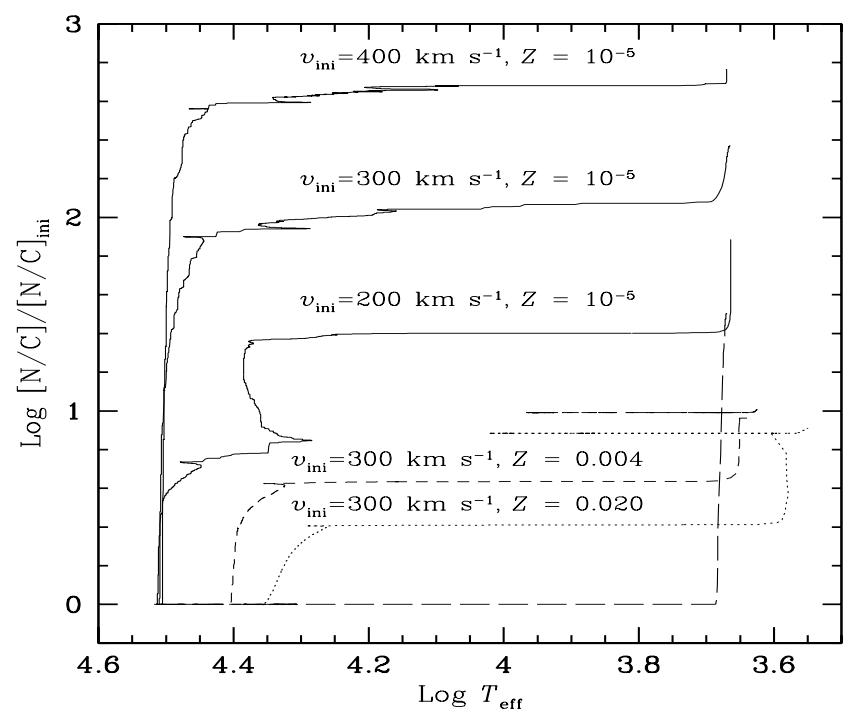

Figure 6. Relative enrichments in [N/C] for models of $9 \mathrm{M}_{\odot}$ with different initial $Z$ and rotational velocities.

its He-burning phase. We emphasize that the velocity of $800 \mathrm{~km} \mathrm{~s}^{-1}$, which may seem high, only corresponds to $\Omega / \Omega_{\mathrm{c}}=0.70$.

\section{Surface enrichments. The case of $\omega$ centauri}

As a consequence of mixing, lower $Z$ stars show larger relative $[N / C]$ enrichments than solar type stars (Fig. 6). As low $Z$ stars also loose mass, their winds with a peculiar composition contribute to the chemical enrichments of galaxies. This may explain the chemical abundances in $\mathrm{C}$-rich extremely metal poor stars (see Meynet et al. these Proceedings).

The globular cluster $\omega$ Centauri has many remarkable properties, in particular the existence of a double Main Sequence (Anderson 1997; see also Bedin 2004). The bluer Main Sequence (bMS) is interpreted in term of a strong excess of helium (Norris 2004), an interpretation well supported by stellar models and studies of the morphology of the horizontal branch stars (Piotto et al. 2005). The challenging problem, however, is that the bluer sequence with a metallicity $[\mathrm{Fe} / \mathrm{H}]=-1.2\left(Z=2 \cdot 10^{-3}\right)$ implies an helium content $Y=0.38$, i.e. an helium enrichment $\Delta Y=0.14$. This in turn demands a relative helium to metal enrichment $\Delta Y / \Delta Z$ of the order of 70 (Piotto et al. 2005; see also Gratton, these Proceedings). This value of $\Delta Y / \Delta Z$ is enormous and more than one order of magnitude larger than the current value of $\Delta Y / \Delta Z=4-5$ (Pagel et al. 1992) obtained from observations of extragalactic HII regions. The low ratio by Pagel et al. is also consistent with the chemical yields from pre-supernovae models forming black holes above about $20-25 \mathrm{M}_{\odot}$ (Maeder 1992). In addition, $\omega$ Centauri shows no carbon excess, i.e. one has $[C / M]=0$ for both the bMS and the red main sequence (rMS). For nitrogen, Piotto et al. find a ratio $[N / M]=1.0-1.5$ of nitrogen to metals for the bMS and $[N / M] \leqslant 1.0$ for the rMS, while for the s-elements, $[B a / M]=0.7$ for the bMS and 0.4 for the rMS.

Various explanations of the peculiar helium content of the bMS stars have been considered (Norris 2004; Piotto et al. 2005). Here we propose another possibility. The winds of 
both massive and AGB stars with rotation produce large yields in helium with $\Delta Y / \Delta Z$ ratios of the order of $10^{2}$, with large $[N / F e]$ excesses. At high rotation velocities in both massive and AGB stars, large $[\mathrm{C} / \mathrm{Fe}]$ and $[\mathrm{O} / \mathrm{Fe}]$ excesses go along with the $\mathrm{N}$-excesses (Meynet, Ekström \& Maeder 2005). The three excesses are of the same size at extreme rotation, while at moderate rotation the $\mathrm{N}$-excesses remain important and the $\mathrm{C}-$ and $\mathrm{O}$-excesses become smaller or even disappear. The same trend occurs for fast rotating models, when moving from $Z=10^{-8}$ to $10^{-3}$. In the AGB models with rotation, there is also a large excess of ${ }^{22} \mathrm{Ne}$ together with excesses of ${ }^{25} \mathrm{Mg}$ and ${ }^{26} \mathrm{Mg}$, implying a production of s-elements. There is no reason why the wind enrichment would be made only by massive stars or only by AGB stars, since contributions from the full mass spectrum may be expected. Pertinent tests on the relative contributions of AGB and massive stars for the He-excess observed in the bMS can be provided by some abundance ratios like $\mathrm{Mg} / \mathrm{Al}, \mathrm{Na} / \mathrm{Mg}, \mathrm{Ne} / \mathrm{N}$ and isotopic ratios such as ${ }^{12} \mathrm{C} /{ }^{13} \mathrm{C},{ }^{16} \mathrm{O} /{ }^{18} \mathrm{O}$ and ${ }^{17} \mathrm{O} /{ }^{18} \mathrm{O}$.

Why is $\omega$ Centauri so unique in having this helium rich bMS ? Likely this massive cluster formed as a starburst and experienced a very fast initial chemical enrichment. The lowest mass stars of the bMS down to at least $0.2 \mathrm{M}_{\odot}$ need about $2 \cdot 10^{8}$ yr to be formed. Thus, all stars with a mass above about $4 \mathrm{M}_{\odot}$ had the time to contribute to the cluster enrichment. The wind ejecta of AGB stars and of red supergiants have velocities of the order of $10^{2} \mathrm{~km} \mathrm{~s}^{-1}$ or less and are likely to be kept in the cluster core. Since $\omega$ Centauri may be the stripped core of an ancient dwarf galaxy feeding the initial Milky Way (Bekki \& Freeman 2003), it would not be surprising that most supernova ejecta at velocities above $10^{4} \mathrm{~km} \mathrm{~s}^{-1}$ also escaped from $\omega$ Centauri (cf. also Norris 2004 and Piotto et al. 2005). The absence of ejecta from SNIa (Cunha et al. 2002), which may originate from stars initially less massive than $8 \mathrm{M}_{\odot}$, is an additional argument for the escape from the cluster core of the supernova ejecta. Thus, we propose that the He-rich blue MS of $\omega$ Centauri results mainly from the peculiar enrichments by the stellar winds of rotating stars in the first stellar generations.

\section{References}

Anderson, J. 1997, Ph. D. thesis, Univ. California, Berkeley

Bedin, L., Piotto, G., Anderson, J. et al. 2004, ApJ 605, L128

Bekki, K. \& Freeman, K.C. 2003, MNRAS 346, L11

Cunha, K., Smith, V.V., Suntzeff, N.B., et al. 2002, AJ 124, 379

Feijoo, J.M. 1999, Internal report, Diploma work, Geneva Observatory

Maeder, A. 1992, A\&SA 264, 105

Maeder, A., Meynet, G. 2001, A\&A 373, 555 (paper VII)

Maeder, A. 2002, $A \mathscr{E} A$ 392, 575

Maeder, A., Grebel, E. Mermilliod, J.C. 1999, A\&A A 1999, 346, 459

Maeder A., Zahn, J.P. 1998, A\&\&A 334, 1000

Meynet G., Maeder A. 2002, A\&A 390, 561 (paper VIII)

Meynet G., Maeder A. 2004, A\& A 429, 581 (Paper XI)

Meynet G., Ekström, S. Maeder A. 2005, $A \mathscr{\xi} A$ in press

Norris, J.E. 2004, ApJ 612, L25

Pagel, B.E.J., Simonson, E.A., Terlevich, R.J., \& Edmunds, M.G. 1992, MNRAS 255, 325

Piotto, G., Villanova, S., \& Bedin, L.R., et al. 2005, ApJ 621, 777

Venn, K., \& Przybilla, N. 2003, in CNO in the Universe, Eds. C. Charbonnel et al. ASP Conf. Ser. 304, p. 20.

Zahn J.P. 1992, A\&A 265, 115 